\title{
MIXED INFECTION BY FOWLPOX VIRUS AND CHLAMYDOPHILA PSITTACI IN A COMMERCIAL LAYING HEN FLOCK
}

\author{
Teresa Agnieszka KARPIŃSKA ${ }^{1,2}$, Wojciech KOZACZYŃSKI ${ }^{1,2}$, Krzysztof NIEMCZUK ${ }^{2}$, \\ Agnieszka JASIK $^{1,2}$, Anna KYCKO ${ }^{1,2}$ and Michał REICHERT ${ }^{1,2^{*}}$ \\ ${ }^{1}$ Department of Pathology, National Veterinary Research Institute, \\ Partyzantów Avenue 57, 24-100 Puławy, Poland; \\ ${ }^{2}$ National Veterinary Research Institute - State Veterinary Institute, Puławy, Poland
}

(Received 26 June 2012; accepted 27 September 2012)

\begin{abstract}
An outbreak of fowlpox occurred in a commercial laying hen flock in one of the western provinces of Poland. Clinical signs suggested fowlpox and the diagnosis was confirmed by histopathological detection of Bollinger bodies within the epithelial cells. Detailed ultrastructural examination revealed an additional concurrent infection with chlamydia-like particles. The particles were identified by PCR as fowlpox virus and Chlamydophila psittaci. It is worth noting that both pathogens can generate morphologic forms capable of prolonged survival and inducing latent and persistent infection. We suggest a possible interaction between the two pathogens on ultrastructural level and assess the clinical consequences of the mixed infection. This study also demonstrates a potential of the transmission electron microscope (TEM) for identifying a superinfection with another pathogen (in this case $C$. psittaci), which may remain undetected by routine techniques.
\end{abstract}

Key words: Fowlpox virus, Chlamydophila psittaci, laying hen, co-infection, electron microscopy, clinical aspects

Avian pox is a slow-spreading infectious disease of domestic and wild birds caused by a DNA virus, member of the genus Avipoxvirus, family Poxviridae (Palade et al., 2008; Tripathy and Reed, 2008). Affected birds develop lesions in the skin as well as in the upper gastrointestinal and respiratory tracts. In poultry, the disease is a result of infection with the fowlpox virus and it manifests itself either as cutaneous (dry pox) or diphtheritic (wet pox) form, or both (Khan et al., 2009; Hess et al., 2011). The cutaneous form is characterised by the presence of papular or ulcerative lesions on the unfeathered skin (Tripathy and Reed, 2008; Cheville and Lehmkuhl, 2009). The diphtheritic form affects the mucous membranes of the mouth, tongue, oesophagus or trachea, with obvious white nodules that coalesce into fibrinous plaques (Shivaprasad et al., 2009). Viruses pre-

*Corresponding author; E-mail: reichert@piwet.pulawy.pl; Phone: 0048 (81) 889-3060; Fax: 0048 (81) 886-2595 
sent in dried scabs are resistant to extreme conditions and can survive for months or even years in the host, providing a source for new infections.

Chlamydophila psittaci (C. psittaci), a member of the family Chlamydiaceae, is an obligate intracellular bacterium pathogenic for humans and animals (Suwa and Itakura, 1992; Beatty et al., 1994; Zhang et al., 2008). It is responsible for respiratory, digestive or systemic infection in birds and can also persist for a very long time in host cells under disadvantageous conditions (Hogan et al., 2004; Andersen and Vanrompay, 2008).

This paper reports the results of an ultrastructural study and analyses, on subcellular level, several aspects of dual infection with fowlpox virus and $C$. psittaci in laying hens. The pathological and clinical implications of the mixed infection are discussed.

\section{Materials and methods}

\section{Sampling}

Heads of six birds with pathological lesions (51-week-old Hy-Line Brown layers) were obtained from an affected flock located in one of the western provinces of Poland.

\section{Histological examination}

Skin samples from head lesions were fixed in $10 \%$ neutral buffered formalin, processed by routine methods, stained with haematoxylin-eosin (HE) and examined using a Zeiss Axioskop 2 light microscope. In parallel, frozen tissue samples were only fixed and sectioned on a freezing microtome, and stained with Sudan IV.

\section{Ultrastructural examination}

Small fragments of refrozen material were fixed with 3\% glutaraldehyde and then $1 \%$ osmic acid, dehydrated in increasing concentrations of ethanol, and embedded in Epon 812. Samples were sliced using a RMC PowerTome XL ultramicrotome fitted with a diamond knife. Sections were stained with methylene blue and examined under a Zeiss Axioskop 2 Plus microscope. Ultrathin sections were contrasted with uranyl acetate and lead citrate, and examined using a Zeiss Libra 120 digital transmission electron microscope (TEM).

\section{Molecular analysis}

DNA was extracted from the frozen material using standard techniques. Oligonucleotide outer primers (191 CHOMP and CHOMP371) used in PCR targeted the ompA gene specific to all Chlamydiaceae and the inner primers (218PSITT and CHOMP336s) targeted C. psittaci (Sachse and Hotzel, 2003). 
Poxvirus-specific DNA was amplified using core protein targeted primers described by Tadese et al. (2007). The PCR products were sequenced and the identity of both amplicons (the $C$. psittaci specific 411-bp and the fowlpox virus specific 578-bp fragment) was confirmed.

\section{Results}

\section{Clinical observations}

The farm owner stated that the affected birds exhibited reduced feed and water consumption and an estimated $15 \%$ drop in egg production. The overall mortality rate was about $20 \%$. The presence of pustules in affected birds was noted on the combs, wattles and in the corners of the beak, while erosions and ulcerations were evident on the tongue and in the beak cavity. The flock was not vaccinated against pox.

\section{Light microscopy}

Histopathological examination revealed different types of changes in the unfeathered skin samples collected from the bird's heads (Fig. 1). In many places a high degree of congestion (C) associated with haemorrhage, signs of oedema, ectatic lymphatics, as well as the presence of lymphocytes, macrophages and heterophils in the form of diffuse or more localised infiltrates were observed (not shown). Occasionally pustules or molluscum ulcerations (U) with clumps of bacteria, degenerate inflammatory cells and cellular and karyorrhectic debris were seen. In many places proliferation and dilatation of the vessels $(\mathrm{V})$ and degeneration of the epidermis (D) occurred (Fig. 1A). Strong multiplication and hypertrophy of epidermal cells with superficial hyperkeratosis was commonly seen. Epidermal cells frequently underwent ballooning degeneration (B) and contained large eosinophilic intracytoplasmic inclusions, Bollinger bodies (B.b) (Fig. 1B). Many necrotic keratinocytes $(\mathrm{N})$ or swollen cells with intracytoplasmic inclusions (B.b and I) and numerous coccoid bacteria (b) were visible after methylene blue staining in the desquamatous stratum corneum (Str.c.) (Fig. 1C). Additional staining of frozen sections with Sudan IV revealed their more compact structure (not shown).

Fig. 1. Histopathological examination of the unfeathered hen's skin lesions. A. Pustule formation and ulceration $(\mathrm{U})$ in the epidermis with many bacterial colonies, degenerate inflammatory cells and cellular and necrotic debris. Proliferation of blood vessels (V), frequently with congestion (C), in the altered skin and degeneration of the epidermis (D). Haematoxylin and eosin (HE) stain, bar $=100 \mu \mathrm{m} . B$. Cytoplasmic vacuolation (ballooning degeneration) $(\mathrm{B})$ of keratinocytes with intracytoplasmic inclusions (Bollinger bodies) $($ B.b $\rightarrow$ ) and dilatation of the lymphatic vessels $(\mathrm{V})$.

HE stain, bar $=20 \mu \mathrm{m}$. $C$. Numerous coccoid bacteria (b) in the desquamatous stratum corneum

(Str.c.) Note abundance of degenerated or necrotic $(\mathrm{N})$ epidermal cells, and hyperplastic

keratinocytes with Bollinger bodies $($ B. $b \rightarrow$ ) or other inclusions $(\mathrm{I} \rightarrow$ ). Methylene blue stain, bar $=20 \mu \mathrm{m}$ 

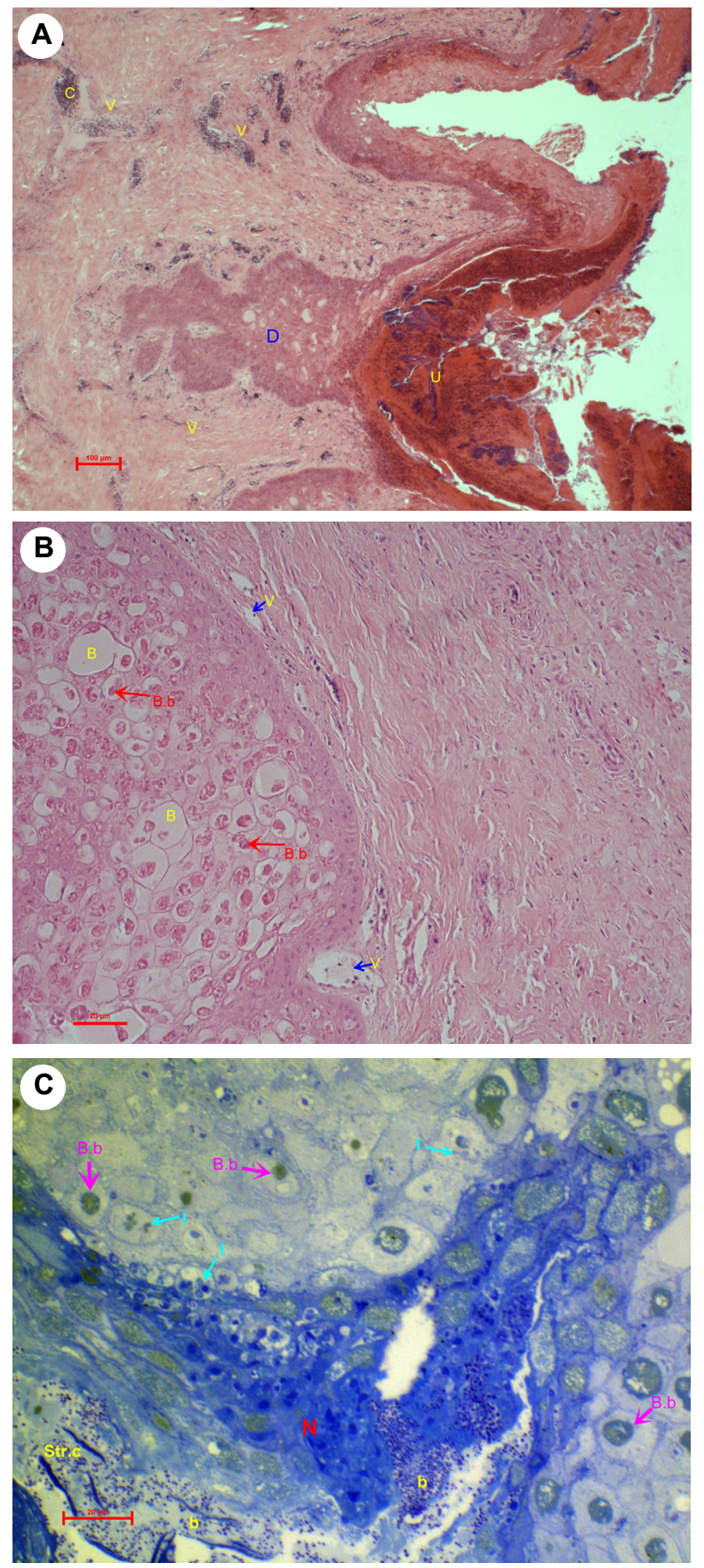


\section{Ultrastructural study}

Electron microscopic analysis showed an existing co-infection with pox-like and chlamydia-like particles (Fig. 2). The former were oval in shape, measured about 179-314 nm and consisted of electron-dense biconcave cores (P.vs). They were surrounded by rodlets (Rs) or lipid droplets (L) and gathered together within intracytoplasmic inclusions - Bollinger bodies (B.b) - inside the keratinocytes (Fig. 2A). The latter were larger and rounded, and were identified on the basis of their size and shape as $C$. psittaci elementary bodies (C.p.EBs) measuring about $509-576 \mathrm{~nm}$ in diameter, $C$. psittaci reticulate bodies (C.p.RBs) measuring about 659-917 nm in diameter and C. psittaci intermediate bodies (C.p.IB) measuring about 590-905 $\mathrm{nm}$ in diameter. They were situated within their own, large or small, intracytoplasmic inclusions (Fig. 2B). Both pathogens (fowlpox virus and C. psittaci) were detected in the neighbouring or in the same keratinocytes (Fig. 2C). Cells containing one pathogen were more numerous than those with both pathogens. Interestingly, the chlamydial particles in the dually infected cells were dispersed in the cytoplasm of these cells without protective vacuoles (Fig. 2C) or, if they were present within small inclusions, they were aberrant, and the infected keratinocytes had degenerative organelles and nuclei with a granulated chromatin. Reticulate bodies of chlamydia in a binary fission form were very sparse in all samples.

\section{Molecular analysis}

The presence of two pathogens in the same specimens was subsequently confirmed by PCR followed by electrophoresis showing the products of expected size.

\section{Discussion}

Mixed infections occur frequently in both humans and animals, and have specific pathological consequences on the course of the disease. Simultaneous infection may involve two or more viruses, for example: fowlpox virus (FWPV) and adeno-like virus, herpesvirus or reticuloendotheliosis virus (Diallo et al., 1998; Singh et al., 2000; Diallo et al., 2010). Mixed infection may also involve viruses and bacteria, for example Staphylococcus hyicus and poxvirus (Devriese et al., 1992), C. trachomatis and herpesvirus (Deka et al., 2006), chlamydia and poxvirus (Jacobson and Telford, 1990) or other combinations, for example E. coli and pulmonary aspergillosis (Kim et al., 2003), C. psittaci, fowlpox virus, Haemophilus gallinarum and Ascaridia galli (Malkinson et al., 1987), an Avipoxvirus member, C. psittaci or fungi (Bailey et al., 2002). There is evidence that coinfection can be beneficial to one or both pathogens (Hament et al., 1999) or, conversely, can inhibit the growth of one or both of them (Heike et al., 1997). 

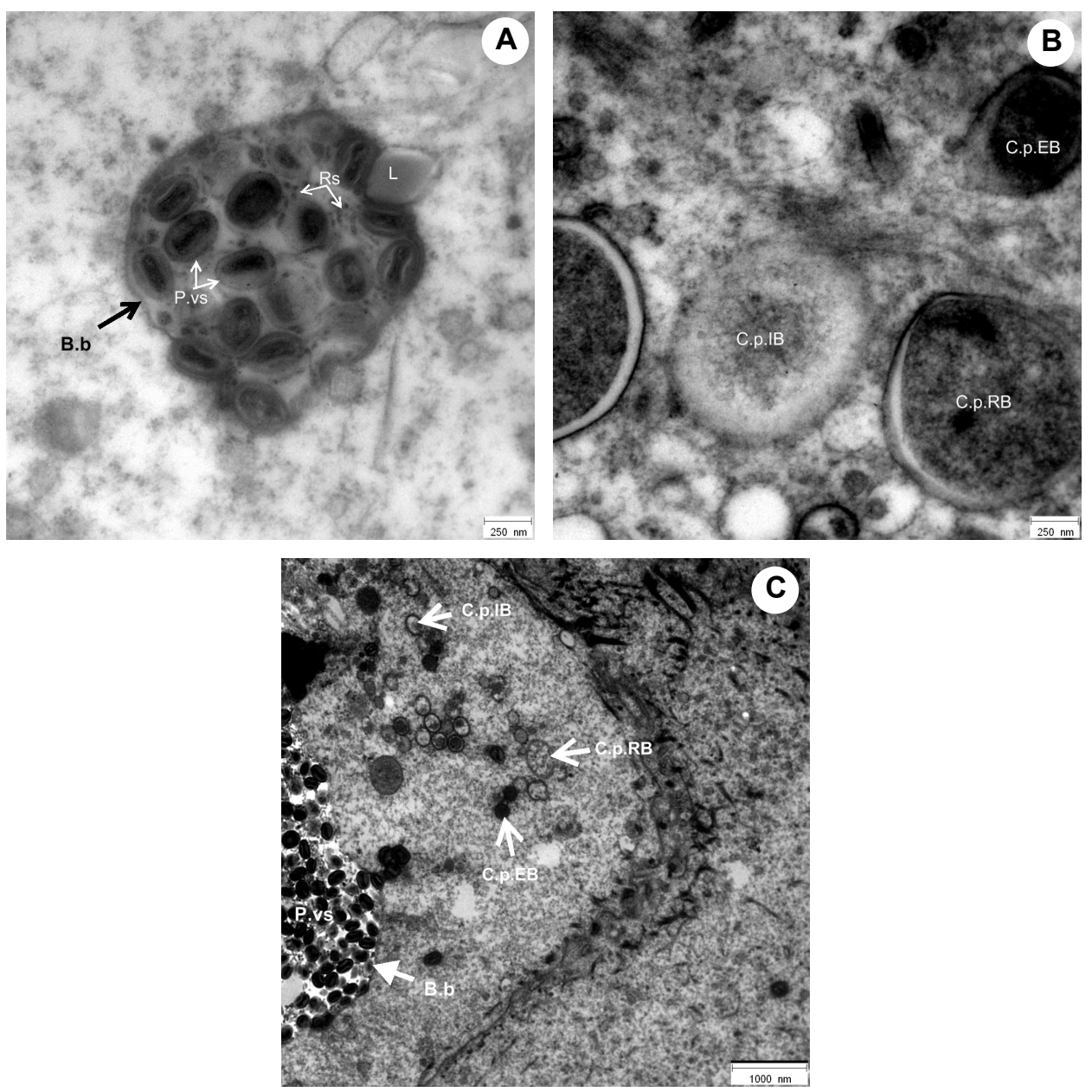

Fig. 2. Ultrastructural examination of the hen's skin. $A$. The Bollinger body $($ B.b $\rightarrow$ ) filled with pox virions (P.vs), rodlets (Rs) and lipid droplet (L). Uranyl acetate and lead citrate stain, bar $=250 \mathrm{~nm} . B$. A fragment of the chlamydial inclusion is filled with elementary (C.p.EB), intermediate (C.p.IB) and reticulate (C.p.RB) bodies of Chlamydophila psittaci within the keratinocyte. Uranyl acetate and lead citrate stain, bar $=250 \mathrm{~nm}$. C. Pox virions (P.vs) within the Bollinger body (B.b), and chlamydial elementary (C.p.EB), reticulate (C.p.RB), intermediate (C.p.IB) bodies dispersed in the cytoplasm of the same prickle cell. Uranyl acetate and lead citrate stain, bar $=1000 \mathrm{~nm}$

The exact mechanism of synergism between bacteria and viruses in the course of co-infection is not known, and various explanations have been proposed. Among them, suppression of the host defence mechanisms, initiation of tissue damage, altered production of inflammatory mediators, modifying organspecific defence mechanisms and encouraging bacterial adherence and growth are the most plausible (Zhang et al., 2008). Hament et al. (1999) emphasised the 
particular importance of bacterial-host cell interactions resulting from the presence of viral proteins in host cells, and differential expression of receptors on the host cell membrane (Beagley et al., 2009). These interactions may augment bacterial adherence during viral infection, adding to the effect of physical damage of the target tissue.

Deka et al. (2006) provided evidence suggesting that the herpes simplex virus co-infection with Chlamydia trachomatis infected cells stimulates the formation of persistent chlamydiae described by Hogan et al. (2004). In this case, the term 'persistent' is used to describe a long-lasting active process (Abdelrahman et al., 2011) involving an atypical form of growth, with the diminished metabolism and reduced production of infectious particles, manifesting as swollen, aberrantly shaped and electron-lucent bacteria. Reticulate bodies of Chlamydia have low metabolism and rarely exhibit binary fission. This is a response to stressful conditions in the host cell. Hogan et al. (2004) described this stage of chlamydia as viable but not cultivable, resulting in a long-term relationship with the infected host cell.

The exact mechanism of this persistence is still unclear (Schoborg, 2011). There are several known inducers of chlamydial persistence, including exposure to interferon-gamma (IFN- $\gamma$ ), tumour necrosis factor alpha (TNF- $\alpha$ ), deprivation of iron, amino acids and glucose, or exposure to antibiotics (Hogan et al., 2004). Deka et al. (2006) could not explain this mechanism, but concluded that coinfection may trigger a novel host pathway and restrict chlamydial development. We did not have the opportunity to perform detailed quantitative analysis on a molecular level to estimate the developmental stage (normal or persistent) of the chlamydia. Analysis of the expression pattern of the non-coding RNAs (ncRNAs) by an intergenic microarray or northern blotting could provide such information (Coles et al., 1993; Abdelrahman et al., 2011). However, our ultrastructural study of necropsy tissue samples can help to clarify this issue.

As shown by electron microscopy, there were numerous keratinocytes with large Bollinger bodies, but cells with intracellular inclusions filled with chlamydial particles were scarce and usually contained single elementary bodies of chlamydia. In our opinion, the most likely scenario in this case is that primary infection was caused by the fowlpox virus, which produced strong epidermal proliferative lesions forming vesicles and pustules that eroded to leave molluscum ulcerations (Cheville and Lehmkuhl, 2009). These lesions weakened the host cell plasma membranes, enabling infection by a second pathogen, in this case $C$. psittaci. It is well known that chlamydiae typically invade mucous epithelial cells, which they penetrate more easily (Hogan et al., 2004; Schoborg, 2011).

Chlamydia is a type of bacterium that needs a healthy host cell with properly formed organelles in order to derive energy from it (Cheville and Lehmkuhl, 2009). Firstly, it must be capable of specifically attaching to the surface of the target cell and then to form its own membrane-lined vacuole created from the 
host cell plasma membrane. This unique structure protects the pathogen from the host cell's lytic enzymes and facilitates the transfer of specific metabolites from the cytoplasm (Beatty et al., 1994). The results of electron microscopic examination suggest that it was easier for chlamydiae to penetrate cell membranes weakened by primary poxvirus infection. The pathogen could enter the Bollinger body, but was unable to create a sufficient protective border. We hypothesise that impairment of the host cell membrane by the virions is beneficial for non-viral pathogens, for example chlamydia, but after entering the cell, viruses need enzymes from this host cell for their own replication. This causes lysis in some cell organelles, which alters cell metabolism and leads to cellular destruction. In our opinion, chlamydial particles present in such cells are prevented from growing and transiting to reticulate bodies, but remain infectious for other target cells (Beatty et al., 1994; Hogan et al., 2004; Schoborg, 2011).

Further studies are required to gain a better understanding of the consequences of mixed infection by fowlpox virus and C. psittaci on the subcellular level. It was also reported that chlamydiae can mediate a switch from an inflammatory to an anti-inflammatory function within host cells due to the downregulation of cytokines like IFN- $\gamma$, resulting in a chronic course of infection (Beatty et al., 1994). Mixed infection of the same host with poxvirus and C. psittaci was reported previously in poultry, but the authors did not analyse the pathogen interplay on the subcellular level (Malkinson et al., 1987).

There is a report on the presence of both poxviral and chlamydial inclusions in the circulating monocytes of a flap-necked chameleon (Jacobson and Telford, 1990). The authors found that at the time of death this lizard exhibited monocytaemia and massive infection of all monocytes with one or both pathogens. The biological consequences of the mixed infection were unclear, but a link between dual infection and the clinical status of the affected animal could not be excluded. Another study provided evidence that chlamydia can infect circulating cells, particularly monocytes, macrophages and dendritic cells and alter their immune functions, promoting their persistence in the host and progression of the disease (Beagley et al., 2009). Our samples were limited to formalin-fixed or frozen head tissues, and we could not examine other types of cells (such as circulating leukocytes) for the presence of similar inclusions.

The long duration of the reported avian pox outbreak (several months) and the high mortality rate (about $20 \%$ ) point to an essential contribution of coinfecting C. psittaci to the pathogenesis and clinical picture of the infection. Pox is usually easily recognisable because of the characteristic clinical signs. However, the yellow-green and gelatinous skin lesions with exudate, present near the beak or eyes, indicate a need to test for chlamydiosis. Additional alarming signs are trembling, imbalanced gait or emaciation. In particular, hypofunction of the ovaries and poor egg production observed in the study can be linked to infection by this second pathogen (Zhang et al., 2008). 
It is worth noting that, independently of the important biological implications of mixed infection by fowlpox virus and C. psittaci, the clinical symptoms of fowlpox can mask or obscure symptoms of chlamydiosis. The consequences of that pose the potential threat of serious (sometimes fatal) C. psittaci infection to poultry workers and their domestic animals (Heike et al., 1997). To prevent poor outcomes, birds should be vaccinated, but an effective vaccine against chlamydiosis is not yet available (Andersen and Vanrompay, 2008). There are, however, good commercial pox vaccines (Prukner-Radovici et al., 2006; Tripathy and Reed, 2008).

Further studies are required to clarify the relationship between the two pathogens in the course of concurrent infection in more detail.

\section{References}

Abdelrahman, Y. M., Rose, A. and Belland, R. J. (2011): Developmental expression of non-coding RNAs in Chlamydia trachomatis during normal and persistent growth. Nucl. Acids Res. 39, $1843-1854$.

Andersen, A. A. and Vanrompay, D. (2008): Avian chlamydiosis (psittacosis, ornithosis). In: Saif, Y. M., Fadly, A. M., Glisson, J. R., McDougald, L. R., Nolan, L. K. and Swayne, D. E. (eds) Diseases of Poultry. 12th edition. Blackwell Publishing, Ames, Iowa. pp. 971-986.

Bailey, T. A., Silvanose, C., Manvell, R., Gough, R. E., Kinne, J., Combreau, O. and Launay, F. (2002): Medical dilemmas associated with rehabilitating confiscated Houbara Bustard (Chlamydotis undulatamacqueenii) after avian pox and paramyxoviruses type 1 infection. J. Wildl. Dis. 38, 518-532.

Beagley, K. W., Huston, W. M., Hansbro, P. M. and Timms, P. (2009): Chlamydial infection of immune cells: altered function and implications for disease. Crit. Rev. Immunol. 29, 275-305.

Beatty, W. L., Morrison, R. P. and Byrne, G. I. (1994): Persistent Chlamydiae: from cell culture to a paradigm for chlamydial pathogenesis. Microbiol. Rev. 58, 686-697.

Cheville, N. F. and Lehmkuhl, H. (2009): Cytopathology of viral diseases. In: Cheville, N. F. (ed.). Ultrastructural Pathology - The Comparative Cellular Basis of Disease. 2nd edition. Wiley-Blackwell, Hoboken, NJ. pp. 318-326.

Coles, A. M., Reynolds, D. J., Harper, A., Devitt, A. and Pearce, J. H. (1993): Low-nutrient induction of abnormal chlamydial development: a novel component of chlamydial pathogenesis? FEMS Microbiol. Lett. 106, 193-200.

Deka, S., Vanover, J., Dessus-Babus, S., Whittimore, J., Howett, M. K., Wyrick, P. B. and Schoborg, R. V. (2006): Chlamydia trachomatis enters a viable but non-cultivable (persistent) state within herpes simplex virus type 2 (HSV-2) co-infected host cells. Cell. Microbiol. 8, $149-162$.

Devriese, L. A., Uyttebroek, E., Dom, P., De Herdt, P., Ducatelle, R. and Haesebrouck, F. (1992): Staphylococcus hyicus associated with pox in chickens and in turkeys. Avian Pathol. 21, 529-533.

Diallo, I. S., Mackenzie, M. A., Spradbrow, P. B. and Robinson, W. F. (1998): Field isolates of fowlpox virus contaminated with reticuloendotheliosis virus. Avian Pathol. 27, 60-66.

Diallo, I. S., Taylor, J., Gibson, J., Hoad, J., de Jong, A., Hewitson, G., Corney, B. G. and Rodwell, B. J. (2010): Diagnosis of a naturally occurring dual infection of layer chickens with fowlpox virus and gallid herpesvirus 1 (infectious laryngotracheitis virus). Avian Pathol. 39, $25-30$. 
Hament, J. M., Kimpen, J. L., Fleer, A. and Wolfs, T. F. (1999): Respiratory viral infection predisposing for bacterial disease: a concise review. FEMS Immunol. Med. Mic. 26, 189-195.

Hess, C., Maegdefrau-Pollan, B., Bilic, I., Liebhart, D., Richter, S., Mitsch, P. and Hess, M. (2011): Outbreak of cutaneous form of poxvirus on a commercial turkey farm caused by the species fowlpox. Avian Dis. 55, 714-718.

Heike, A., Holcinger-Umlauf, M., Marschang, R. E., Gravendyck, M. and Kaleta, E. F. (1997): Investigation on the frequency of Chlamydia sp. infections in tits (Paridae). Avian Pathol. 26, 779-789.

Hogan, R. J., Mathews, S. A., Mukhopadhyay, S., Summergill, J. T. and Timms, P. (2004): Chlamydial persistence: beyond the biphasic paradigm. Infect. Immun. 72, 1843-1855.

Jacobson, E. R. and Telford, S. R. (1990): Chlamydial and poxvirus infections of circulating monocytes of a flap-necked chameleon (Chamaeleo dilepis). J. Wildl. Dis. 26, 572-577.

Khan, A., Yousaf, A., Khan, M. Z., Siddique, M., Gul, S. T. and Mahmood, F. (2009): Cutaneous form of pox infection among captive peafowl (Pavo cristatus) chicks. Avian Pathol. 38, 65-70.

Kim, T. J., Schnitzlein, W. M., McAloose, D., Pessier, A. P. and Tripathy, D. N. (2003): Characterization of an avianpox virus isolated from an Andean condor (Vultur gryphus). Vet. Microbiol. 96, 237-246.

Malkinson, M., Machany, S., Aronovici, A., Davidov, K. and Weisman, Y. (1987): Mixed infection with Chlamydophila psittaci, fowlpox virus and Haemophilus gallinarium in broiler breeder chicks. Vet. Rec. 120, 461-462.

Palade, E. A., Biró, N., Dobos-Kovács, M., Demeter, Z., Mándoki, M. and Rusvai, M. (2008): Poxvirus infection in Hungarian great tits (Parus major): case report. Acta Vet. Hung. 56, 539-546.

Prukner-Radovici, E., Lüschow, D., Grozdanic, I. C., Tisljar, M., Mazija, H., Vranesic, D. and Hafez, H. M. (2006): Isolation and molecular biological investigation of avian poxvirus from chickens, a turkey, and a pigeon in Croatia. Avian Dis. 50, 440-444.

Sachse, K. and Hotzel, H. (2003): Detection and differentiation of chlamydiae by nested PCR. Methods Mol. Biol. 216, 123-136.

Schettler, E., Fickel, J., Hotzel, H., Sachse, K., Streich, W. J., Wittstatt, U. and Frolich, K. (2003): Newcastle Disease Virus and Chlamydia psittaci in free-living raptors from Eastern Germany. J. Wildl. Dis. 39, 57-63.

Schoborg, R. V. (2011): Chlamydia persistence - a tool to dissect chlamydia-host interactions. Microbes Infect. 13, 649-662.

Shivaprasad, H. L., Kim, T., Tripathy, D., Woolcock, P. R. and Uzal, F. (2009): Unusual pathology of canary poxvirus infection associated with high mortality in young and adult breeder canaries (Serinus canaria). Avian Pathol. 38, 311-316.

Singh, P., Kim, T. J. and Tripathy, D. N. (2000): Re-emerging fowlpox: evaluation of isolates from vaccinated flocks. Avian Pathol. 29, 449-455.

Suwa, T. and Itakura, C. (1992): Ultrastructural studies of chlamydia-infected air sacs of chicks. Avian Pathol. 21, 443-452.

Tadese, T., Potter, A. E., Fitzgerald, S. and Reed, W. M. (2007): Concurrent infection in chickens with fowlpox virus and infectious laryngotracheitis virus as detected by immunohistochemistry and a multiplex polymerase chain reaction technique. Avian Dis. 51, 719-724.

Tripathy, D. N. and Reed, W. M. (2008): Pox. In: Saif, Y. M., Fadly, A. M., Glisson, J. R., McDougald, L. R., Nolan, L. K. and Swayne, D. E. (eds) Diseases of Poultry. 12th edition. Blackwell Publishing, Ames, Iowa. pp. 291-307.

Zhang, F., Li, S., Yang, J., Pang, W., Yang, L. and He, C. (2008): Isolation and characterization of Chlamydophila psittaci isolated from laying hens with cystic oviducts. Avian Dis. 52, 74-78. 